\title{
NOTE ON GENERALIZATION OF THE JENSEN-MERCER INEQUALITY BY TAYLOR'S POLYNOMIAL
}

\author{
ANITA MATKOVIĆ AND JOSIP PEČARIĆ
}

Abstract. We present generalizations of the Jensen-Mercer inequality for the class of $n$-convex functions. The results are obtained by using Taylor's polynomial and four types of Green's functions.

Mathematics subject classification (2010): 26D15, 26D20.

Keywords and phrases: Jensen-Mercer inequality, $n$-convex functions.

\section{REFERENCES}

[1] J. Barić, A. Matković, Bounds for the normalized Jensen-Mercer functional, J. Math. Inequal. 3 (4) (2009), 529-541.

[2] S. I. Butt, K. A. Khan, J. PeČArić, Popovicu type inequalities via Green function and generalized Montgomery identity, Math. Inequal. Appl. 18(4) (2015), 1519-1538.

[3] J. JAKŠETIĆ, J. PEČARIĆ, Exponential convexity method, J. Convex Anal. 20 (1) (2013), 181-197.

[4] J. JAKŠETIĆ, J. PEČARIĆ, A. PERUŠIĆ, Steffensen inequality, higher order convexity and exponential convexity, Rend. Circ. Mat. Palermo. 63(1) (2014), 109-127.

[5] M. Adil Khan, N. Latif And J. PeČARIĆ, Generalization of majorization theorem, J. Math. Inequal. 9 (3) (2015), 847-872.

[6] A. Matković, Generalization of the Jensen-Mercer inequality by Taylor's polynomial, Math. Inequal. Appl. 19(4) (2016), 1387-1398.

[7] T. Popoviciu, Les Fonctions Convexes, Hermann, Paris, 1944.

[8] J. E. Pečarić, F. Proschan and Y. L. Tong, Convex Functions, Partial Orderings, and Statistical Applications, Academic Press, Inc. 1992. 